\title{
Cosmic Bell Test: Measurement Settings from Milky Way Stars
}

\author{
Johannes Handsteiner, ${ }^{1, *}$ Andrew S. Friedman, ${ }^{2, \dagger}$ Dominik Rauch, ${ }^{1}$ Jason Gallicchio, ${ }^{3}$ \\ Bo Liu, ${ }^{1,4}$ Hannes Hosp,${ }^{1}$ Johannes Kofler, ${ }^{5}$ David Bricher, ${ }^{1}$ Matthias Fink, ${ }^{1}$ Calvin Leung, ${ }^{3}$ \\ Anthony Mark, ${ }^{2}$ Hien T. Nguyen,${ }^{6}$ Isabella Sanders ${ }^{2}$ Fabian Steinlechner, ${ }^{1}$ Rupert Ursin, ${ }^{1,7}$ \\ Sören Wengerowsky, ${ }^{1}$ Alan H. Guth, ${ }^{2}$ David I. Kaiser, ${ }^{2}$ \\ Thomas Scheidl, ${ }^{1}$ and Anton Zeilinger ${ }^{1,7,}$ \\ ${ }^{1}$ Institute for Quantum Optics and Quantum Information (IQOQI), Austrian Academy of Sciences, \\ Boltzmanngasse 3, 1090 Vienna, Austria \\ ${ }^{2}$ Department of Physics, Massachusetts Institute of Technology, Cambridge, Massachusetts 02139, USA \\ ${ }^{3}$ Department of Physics, Harvey Mudd College, Claremont, California 91711, USA \\ ${ }^{4}$ School of Computer, NUDT, 410073 Changsha, China \\ ${ }^{5}$ Max Planck Institute of Quantum Optics, Hans-Kopfermann-Straße 1, 85748 Garching, Germany \\ ${ }^{6}$ NASA Jet Propulsion Laboratory, Pasadena, California 91109, USA \\ ${ }^{7}$ Vienna Center for Quantum Science \& Technology (VCQ), Faculty of Physics, \\ University of Vienna, Boltzmanngasse 5, 1090 Vienna, Austria
}

(Received 21 November 2016; revised manuscript received 13 January 2017; published 7 February 2017)

Bell's theorem states that some predictions of quantum mechanics cannot be reproduced by a localrealist theory. That conflict is expressed by Bell's inequality, which is usually derived under the assumption that there are no statistical correlations between the choices of measurement settings and anything else that can causally affect the measurement outcomes. In previous experiments, this "freedom of choice" was addressed by ensuring that selection of measurement settings via conventional "quantum random number generators" was spacelike separated from the entangled particle creation. This, however, left open the possibility that an unknown cause affected both the setting choices and measurement outcomes as recently as mere microseconds before each experimental trial. Here we report on a new experimental test of Bell's inequality that, for the first time, uses distant astronomical sources as "cosmic setting generators." In our tests with polarization-entangled photons, measurement settings were chosen using real-time observations of Milky Way stars while simultaneously ensuring locality. Assuming fair sampling for all detected photons, and that each stellar photon's color was set at emission, we observe statistically significant $\gtrsim 7.31 \sigma$ and $\gtrsim 11.93 \sigma$ violations of Bell's inequality with estimated $p$ values of $\lesssim 1.8 \times 10^{-13}$ and $\lesssim 4.0 \times 10^{-33}$, respectively, thereby pushing back by $\sim 600$ years the most recent time by which any local-realist influences could have engineered the observed Bell violation.

DOI: 10.1103/PhysRevLett.118.060401

In this Letter, we report on a new experimental test of Bell's inequality that, for the first time, uses distant astronomical sources to choose measurement settings. This is the first in a series of "cosmic Bell tests" that will use progressively more distant sources, ultimately pushing the measurement settings' origin to greater and greater cosmological distances [1].

Background.-Scientists have struggled with the alleged incompatibility of quantum entanglement and our everyday intuitions about the physical world since the seminal paper by Einstein, Podolsky, and Rosen (EPR) in 1935 [2]. EPR concluded that the description of reality given by the

Published by the American Physical Society under the terms of the Creative Commons Attribution 4.0 International license. Further distribution of this work must maintain attribution to the author(s) and the published article's title, journal citation, and DOI. quantum-mechanical wave function is incomplete because it is incompatible with the concepts of locality (no physical influences can travel faster than the speed of light in vacuum) and realism (objects possess complete sets of properties on their own, prior to measurement). A wellknown "tool" to experimentally distinguish between the quantum predictions and local-realist alternatives of the sort envisaged by EPR is provided by the famous inequality derived by John Bell in 1964 [3]. Assuming locality and realism, Bell's inequality limits the degree to which measurement outcomes on pairs of distant systems may be correlated, if the measurements of one system are carried out with only limited information about the other. By contrast, measurements on entangled particle pairs in the quantum singlet state, for example, are predicted to violate Bell's inequality. Beginning with Ref. [4], essentially all significant experimental Bell tests to date have supported the quantum-mechanical predictions. 
However, the conclusions of any experiment are valid only given certain assumptions, the violation of which leaves open "loopholes," whereby a local-realist description of nature could still be compatible with the experimental results. (For extensive reviews of Bell-test loopholes, see Refs. [5-7].) For example, the locality loophole concerns whether any information about one side's measurement setting or measurement outcome could have been communicated (at or below the speed of light) to the other side prior to its measurement. This loophole has been closed by spacelike separating measurement-setting choices on each side from the other side's measurement outcomes [8,9]. The fairsampling loophole [10] concerns whether the set of entangled particles detected on both sides was representative of all emitted pairs rather than a biased subensemble, and has recently been closed by ensuring a sufficient total fraction of detected pairs [11-16]. Even more recently, several cuttingedge experiments have demonstrated violations of Bell's inequality while closing both the locality and fair-sampling loopholes simultaneously [17-21].

A third major loophole, known variously as the freedom-of-choice, measurement-independence, or settingindependence loophole [22-25], concerns the choice of measurement settings. In particular, the derivation of Bell's inequality explicitly assumes that there is no statistical correlation between the choices of measurement settings and anything else that causally affects both measurement outcomes. Bell himself observed forty years ago that, "It has been assumed that the settings of instruments are in some sense free variables-say at the whim of experimenters-or in any case not determined in the overlap of the backward light cones" [22]. Recent theoretical work has demonstrated that models that relax this assumption, allowing for a modest correlation between the joint measurement settings and any causal influence on the measurement outcomes, can reproduce the quantum correlations [26-36]. (See also Ref. [37] on some subtleties of addressing the freedom-of-choice loophole.)

Even if nature does not exploit this loophole, testing it experimentally (e.g., Refs. [38,39]) has significant practical relevance for device-independent quantum key distribution [40-42] as well as random-number generation and randomness expansion $[32,43,44]$. In particular, a sophisticated adversary could undermine a variety of quantum information schemes by utilizing the freedom-of-choice loophole $[26,30,36]$.

To the extent that recent experiments have addressed freedom of choice, they have adopted the additional, strong assumption that the relevant causal influences (or "hidden variables") originate together with the entangled particles and hence cannot influence setting choices in spacelike separated regions $[18,19,38]$, or assumed that the settinggeneration process is completely independent of its past $[17,20,21]$. Yet nowhere in the derivation of Bell's inequality does the formalism make any stipulation about where or when such hidden variables could be created or become relevant. In fact, as Bell himself emphasized [22,24] (see also Refs. [1,32]), they could be associated with any events within the experiment's past light cone [45]. Thus, in principle, the possibility exists that the purportedly random setting choices in previous experiments could have been influenced by some unknown cause in their past, exploiting the freedom-of-choice loophole through events as recent as a few microseconds before the measurements [48].

Here we report on an experimental Bell test with polarization entangled photons that, assuming fair sampling, significantly constrains the space-time region from which any such unknown, causal influences could have affected both the measurement settings and outcomes. While simultaneously closing the locality loophole, measurement settings are determined by "cosmic setting generators," using the color of photons detected during real-time astronomical observations of distant stars. We observed Bell violations with high statistical significance and thus conclude that any hidden causal influences that could have exploited the freedom-of-choice loophole would have to have originated from remote space-time events at least several hundred years ago, at locations seemingly unrelated to the entangled-pair creation. Compared to previous experiments, this pushes back by $\sim 16$ orders of magnitude the most recent time by which any local-realist influences could have engineered the observed correlations.

The idea to address freedom of choice by using distant astronomical sources to choose Bell-test measurement settings was already discussed as far back as the 1976 Erice meeting organized by John Bell and Bernard d'Espagnat [49]. While others have also briefly noted this basic premise [38,50,51], this work is the first to implement it experimentally, building on a detailed feasibility study [1].

Experimental implementation.-Figure 1 shows our three experimental sites across Vienna. A central entangled photon source $S$ was located in a laboratory on the 4th floor of the Institute for Quantum Optics and Quantum Information (IQOQI) and the two observers, Alice $(A)$ and Bob $(B)$, were situated on the 9th floor of the Austrian National Bank (OENB) and on the 5th floor of the University of Natural Resources and Life Sciences (BOKU), respectively.

The entangled photon source is based on a Sagnac interferometer $[52,53]$ generating polarization-entangled photon pairs in the maximally entangled singlet state $\left|\Psi^{-}\right\rangle=(1 / \sqrt{2})\left(\left|H_{A} V_{B}\right\rangle-\left|V_{A} H_{B}\right\rangle\right)$. Using single-mode fibers, each entangled photon was guided to an entangled photon transmitting telescope (Tx-EP) located at the rooftop of IQOQI, which sent the photons via free-space quantum channels to Alice and Bob, respectively. Measurement stations for Alice and Bob each featured an entangled photon receiving telescope (Rx-EP), a polarization analyzer (POL), stellar photon receiving telescope 


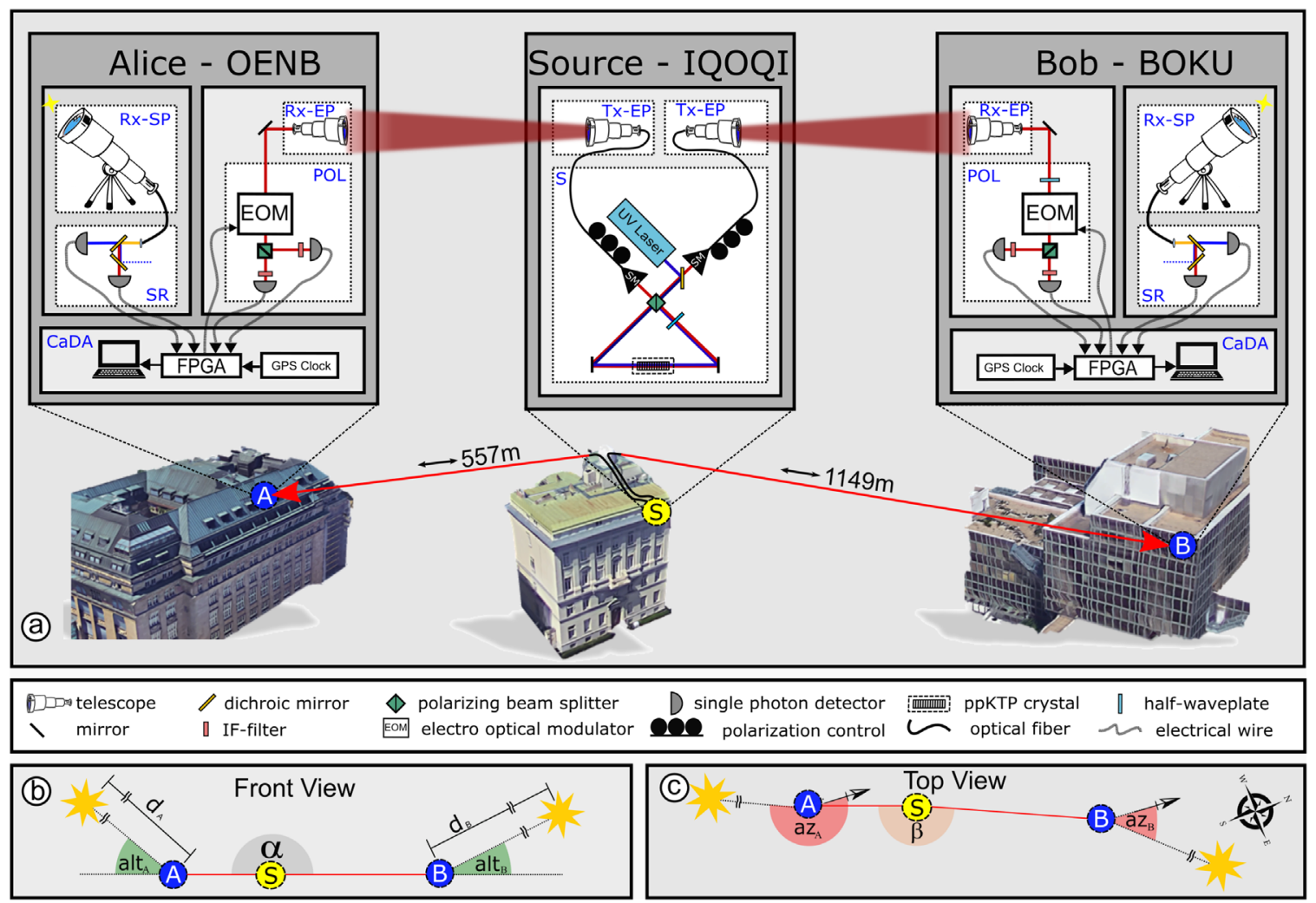

FIG. 1. (a) The three experimental stations and related acronyms are described in the main text. Stellar photon receiving telescopes (Rx-SP) with primary mirror diameters of $0.2032 \mathrm{~m}$ (Meade 8-inch LX200 ACF, $f=2 \mathrm{~m}$ ) and $0.254 \mathrm{~m}$ (Meade 10-inch LX600 ACF, $f=2.032 \mathrm{~m}$ ) were used by Alice and Bob, respectively, although the telescope apertures were each partially covered to limit sky noise. Diameters and focal lengths of the quantum channel telescopes are Alice (Tx-EP: $d=50.8$, $f=100 \mathrm{~mm}$; Rx-EP: $d=80, f=400 \mathrm{~mm}$ ) and Bob (Tx-EP: $d=70, f=280 \mathrm{~mm}$; Rx-EP: $d=140, f=420 \mathrm{~mm}$ ). Latitude, longitude, and elevation for the three experimental sites are Alice $\left(A: 48.21645^{\circ}, 16.354311^{\circ}, 215.0 \mathrm{~m}\right)$, Bob $\left(B: 48.23160^{\circ}\right.$, $\left.16.3579553^{\circ}, 200.0 \mathrm{~m}\right)$, and the Source $\left(S: 48.221311^{\circ}, 16.356439^{\circ}, 205.0 \mathrm{~m}\right)$. (b) and (c) For experimental run 1, the setup deviated from the ideal, colinear 1D case by the angles displayed. Site coordinates yield $\alpha \approx 180^{\circ}$ and $\beta \approx 169^{\circ}$ for both runs. See Table I for the azimuth (az) and altitude (alt) of each star observed during each experimental run. (3D graphics taken from Google Earth, 2016.)

(Rx-SP), a setting reader (SR) and a control and data acquisition unit (CaDA). The entangled photons were collected with the Rx-EP and guided to the polarization analyzer where an electro-optical modulator (EOM) allowed for fast switching between complementary measurement bases. This was followed by a polarizing beam splitter with a single-photon avalanche diode (SPAD) detector in each output port.

The Rx-SPs collected stellar photons, which were guided by multimode fibers to setting readers, where dichroic mirrors with $\sim 700 \mathrm{~nm}$ cutoffs split them into "blue" and "red" arms, each fed to a SPAD. An FPGA board processed the SPAD signals to electronically implement the corresponding EOM measurement setting. Every detector click in a red or blue arm induced a measurement in the following linear polarization bases for Alice: $45^{\circ} / 135^{\circ}$ (blue) and $0^{\circ} / 90^{\circ}$ (red), and Bob: $22.5^{\circ} / 112.5^{\circ}$ (blue) and $-22.5^{\circ} / 67.5^{\circ}$ (red), respectively. Finally, using a GPS-disciplined clock, all SPAD detections from the polarization analyzer and the setting reader were time stamped by the FPGA board and recorded by a computer.

We specifically use photon color to implement measurement settings under the assumption that the wavelength of the photon emitted from the star was determined at the time of emission and unaltered since. Astrophysical motivations for this assumption include the absence of any known mechanism that preferentially reradiates photons at a different wavelength along our line of sight; any such process would violate the conservation of energy and momentum. In addition, the effects of wavelength-dependent attenuation by the interstellar medium are negligible for Milky Way stars within a few thousand light years (ly) [54-56]. By contrast, significant attenuation from the Earth's atmosphere (38\%-45\% loss) as well as from the experimental setup (59\%-61\% loss) is unavoidable (see the Supplemental Material [57]). Thus, our approach requires the assumption that this represents a fair sample of the celestial emissions. 


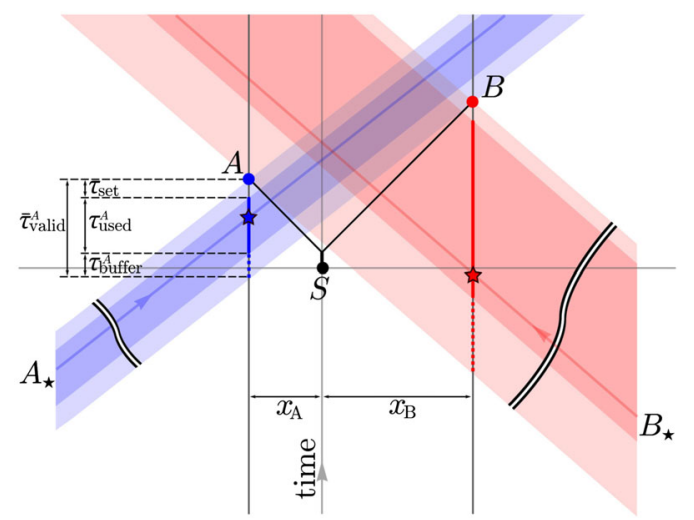

FIG. 2. $(1+1) \mathrm{D}$ space-time diagram for run 1 , with the origin at the entangled pair creation (black dot) and a spatial projection axis chosen to minimize its distance to Alice and Bob. After a fiber delay (thick black line), entangled photons are sent via freespace channels (thin black lines) to be measured by Alice and Bob at events $A$ and $B$. Blue and red stars indicate example valid settings from measuring stellar photons emitted far away at space-time events $A_{\star}$ and $B_{\star}$ (see Fig. 3). Ensuring locality limits valid settings to the shaded regions. Delays to implement each setting and an added safety buffer shorten the validity time windows actually used to the darker shaded regions.

Two other major effects must be considered to account for SPAD detections which do not represent stellar photons with correctly identified colors. First, local sources of noise, including sky glow, light pollution, and dark counts determine between $1 \%$ and $5 \%$ of the settings, as measured by looking at a dark patch of sky next to each star. Only a tenth of these are detector dark counts. In addition, due to imperfect dichroic mirrors, a certain fraction of detected stellar photons will be assigned the wrong setting. Hence, to precisely quantify the full optical path of our settings implementation method, we measured transmission and reflection spectra for the dichroic mirrors, and multiplied this by the spectra of the stars, atmospheric transmission [61], reflection and transmission of optical elements, and the SPAD detector response. Conservative estimates of the fraction of incorrectly determined measurement settings range from $f_{w} \approx 1.4 \%-2.0 \%$, depending on the stellar spectrum and red and blue output port (see the Supplemental Material [57]).

Space-time arrangement.-Ensuring locality requires that any information leaving Alice's star along with her setting-determining stellar photon and traveling at the speed of light could not have reached Bob before his measurement of the entangled photon is completed, and vice versa. This also achieves a necessary condition for freedom of choice.

The projected $(1+1) \mathrm{D}$ space-time diagram in Fig. 2 shows run 1 of our experiment. Entangled photons are generated at point $S$, which coincides with the origin. After local fiber transmission to the transmitting telescopes, which takes a time of $\tau_{\text {fiber }} \approx 180 \mathrm{~ns}$, they are sent via free-space channels to Alice at a projected distance of $x_{A}=557 \mathrm{~m}$ and to Bob at $x_{B}=1149 \mathrm{~m}$ and measured at events $A$ and $B$, respectively. Settings are determined far away at the stellar emission events $A_{\star}$ and $B_{\star}$, respectively. To close the locality loophole, entangled photon detections cannot be accepted outside a certain (maximal) time interval $\tau_{\text {valid }}^{k}(k=\{A, B\})$ after the detection of a stellar photon, which, in general, must be chosen such that the corresponding setting is still space-like separated from the measurement on the other side.

The time-dependent locations of the stars on the sky relative to our ground-based experimental sites make $\tau_{\text {valid }}^{k}(t)$ time-dependent parameters. However, since our Rx-SPs pointed out of windows, resulting in highly restrictive azimuth and altitude limits for the star selection, $\tau_{\text {valid }}^{k}(t)$ did not change significantly during the $3 \mathrm{~min}$ of measurement for each experimental run. Consequently, using spatial site coordinates from Fig. 1 and the star's celestial coordinates (see the Supplemental Material [57]), $\bar{\tau}_{\text {valid }}^{k}=\min _{t}\left\{\tau_{\text {valid }}^{k}(t)\right\}$ was calculated as $2.55 \mu$ s for Alice and $6.93 \mu \mathrm{s}$ for Bob for run 1 (see Table I).

The final time-window $\tau_{\text {used }}^{k}$ was chosen to be as large as possible (see Fig. 2), while also subtracting the time it takes to implement a setting ( $\left.\tau_{\text {set }} \approx 170 \mathrm{~ns}\right)$ and to ensure optimal operation of the EOM (e.g., by minimizing piezoelectric ringing). The latter required subtracting safety buffers of $\tau_{\text {buffer }}^{A}=0.38 \mu$ s for Alice and $\tau_{\text {buffer }}^{B}=1.76 \mu$ s for Bob,

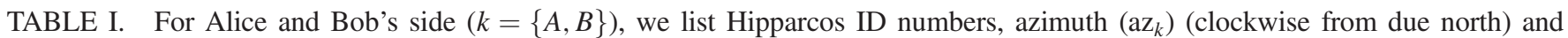
altitude $\left(\right.$ alt $\left._{k}\right)$ above horizon during the observation, and parallax distances $\left(d_{k}\right)$ with errors $\left(\sigma_{d_{k}}\right)$ for stars observed during runs 1 and 2 , which began at UTC 2016-04-21 21:23:00 and 2016-04-22 00:49:00, respectively, each lasting $179 \mathrm{~s} . \bar{\tau}_{\text {valid }}^{k}$ is the minimum time that detector settings are valid while the star on side $k$ remained visible during each run, before subtracting delays and safety margins (see Fig. 2). The last 3 columns show the measured CHSH parameter for runs 1 and 2, as well as the $p$ value and the number of standard deviations $\nu$ by which our local-realist model can be rejected (see the Supplemental Material [57]).

\begin{tabular}{lccrcccccc}
\hline \hline Run & Side & HIP ID & $\mathrm{az}_{k}^{\circ}$ & alt $_{k}^{\circ}$ & $d_{k} \pm \sigma_{d_{k}}[\mathrm{ly}]$ & $\bar{\tau}_{\text {valid }}^{k}[\mu \mathrm{s}]$ & $S_{\text {exp }}$ & $p$-value & $\nu$ \\
\hline 1 & $A$ & 56127 & 199 & 37 & $604 \pm 35$ & 2.55 & 2.43 & $1.8 \times 10^{-13}$ & 7.3 \\
& $B$ & $105259 \mathrm{~A}$ & 25 & 24 & $1930 \pm 605$ & 6.93 & & & \\
2 & $A$ & 80620 & 171 & 34 & $577 \pm 40$ & 2.58 & 2.50 & $4.0 \times 10^{-33}$ & 11.9 \\
& $B$ & 2876 & 25 & 26 & $3624 \pm 1370$ & 6.85 & & & \\
\hline \hline
\end{tabular}




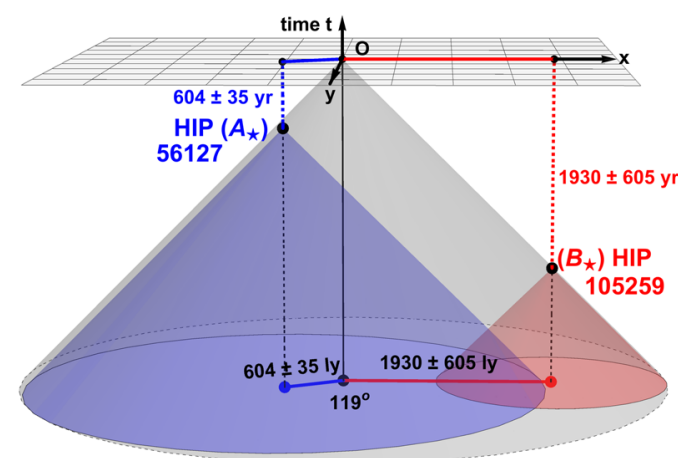

FIG. 3. $(2+1) \mathrm{D}$ space-time diagram for run 1 with past light cones for stellar emission events $A_{\star}$ and $B_{\star}$. Two spatial dimensions are shown ( $x-y$ plane) with the third suppressed. The stellar pair's angular separation on the sky is the angle between the red and blue vectors. Our data rule out local-realist models with hidden variables in the gray space-time region. We do not rule out models with hidden variables in the past light cones for events $A_{\star}$ (blue), $B_{\star}$ (red), or their overlap (purple).

which easily accounted for the delay of stellar photons due to the index of refraction of the atmosphere $\left(\tau_{\text {atm }} \approx 18 \mathrm{~ns}\right)$ [62], and any small inaccuracies in timing or the distances between the experimental stations (see the Supplemental Material [57]).

In Fig. 2, stellar photons arriving parallel to the arrows in the blue and red shaded space-time regions (corresponding to the time intervals $\bar{\tau}_{\text {valid }}^{k}$ ) provide valid basis settings, ensuring spacelike separation for all relevant events. The darker shading corresponds to regions actually used in run 1, with $\tau_{\text {used }}^{A}=2 \mu$ s and $\tau_{\text {used }}^{B}=5 \mu \mathrm{s}$. In run 1, the fractions of time with valid settings for Alice and Bob were $24.9 \%$ and $40.6 \%$, respectively, while in run 2 they were $22.0 \%$ and $44.6 \%$, respectively. Duty cycles for each observer differ primarily due to different values of $\tau_{\text {used }}^{k}$ and different count rates for each star (see the Supplemental Material [57]).

We preselected candidate stars within the highly restrictive azimuth and altitude limits of the stellar photon receiving telescopes from the Hipparcos catalogue $[63,64]$ with parallax distances greater than $500 \mathrm{ly}$, distance errors less than $50 \%$ and Hipparcos $H_{p}$ magnitude between 5 and 9. Combined with the geometric configuration of the sites, selection of these stars ensured sufficient setting validity times on both sides during each experimental run of $179 \mathrm{~s}$. To ensure a sufficiently high signal-to-noise ratio, we chose 5-6 magnitude stars (see the Supplemental Material [57]). Note that to avoid detector saturation, parts of the entrance aperture of the Rx-SPs had to be covered.

Figure 3 shows a $(2+1) \mathrm{D}$ space-time diagram for the stellar emission events from run 1. Events associated with relevant hidden variables could lie within the past light cones of stellar emission events $A_{\star}$ or $B_{\star}$, the most recent of which originated $604 \pm 35$ years ago, accounting for parallax distance errors arising primarily from the angular resolution limits of the Hipparcos mission [63,64].

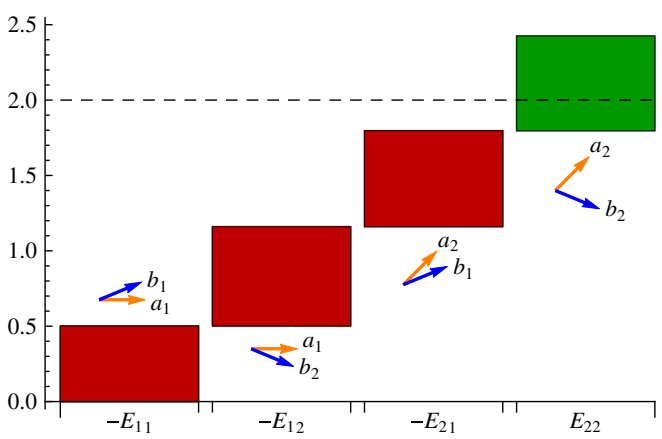

FIG. 4. For run 1, bars show the correlation $E_{i j}$ for each joint setting combination $\left(a_{i}, b_{j}\right)$. CHSH terms are displayed with negative signs (red) and a positive sign (green), showing that our data violate the local-realist bound (dashed line, $S=2$ ). The $\left|E_{i j}\right|$ values are unequal due to limited state visibility and imperfect alignment of the polarization measurement bases.

Analysis and results.-We performed two cosmic Bell tests, each lasting 179 seconds. In runs 1 and 2, Alice and Bob's settings were chosen with photons from Hipparcos stars in Table I. To analyze the data, we make the assumptions of fair sampling and fair coincidences [65]. Thus, all data can be postselected to include coincidence events between Alice's and Bob's measurement stations. We correct for GPS clock drift as in Ref. [66] and identify coincidences within a $2.5 \mathrm{~ns}$ time window.

We then analyze correlations between measurement outcomes $A, B \in\{+1,-1\}$ for particular setting choices $\left(a_{i}, b_{j}\right), i, j \in\{1,2\}$ using the Clauser-Horne-ShimonyHolt (CHSH) inequality [67]:

$$
S \equiv\left|E_{11}+E_{12}+E_{21}-E_{22}\right| \leq 2,
$$

where $E_{i j}=2 p\left(A=B \mid a_{i} b_{j}\right)-1$ and $p\left(A=B \mid a_{i} b_{j}\right)$ is the probability that Alice and Bob measure the same outcome given joint settings $\left(a_{i}, b_{j}\right)$. While the local-realist bound is 2 , the quantum bound is $2 \sqrt{2}$, and the logical (algebraic) bound is 4 . Our run 1 data yield $S_{\exp }=2.425$, while for run 2 , we observe $S_{\exp }=2.502$. Both runs therefore violate the corresponding local-realist bound. See Fig. 4 and Table I.

Our analysis must further consider that some experimental trials will have "corrupt" settings triggered not by genuine stellar photons, but by atmospheric airglow, thermal dark counts, errant dichroic mirror reflections, or other noise in our detectors. Since these events originate very recently in the experiments' past light cone, settings chosen with them are no better (and no worse) than settings chosen with conventional random number generators.

To constrain the fraction of experimental runs we can tolerate in which either or both sides were triggered by a corrupt event, we conservatively assume that any such events can produce maximal CHSH correlations $(S=4)$ [68], whereas settings triggered by correctly identified stellar photons are assumed to obey local realism 
$(S \leq 2)$. An optimally efficient local hidden-variable model would only need to use individual corrupt photons on a single side to achieve $S=4$, without needing to "waste" simultaneous corruptions or events in which changing the path of a stellar photon through the setting reader is unnecessary. We, therefore, use this maximally conservative model as our null hypothesis.

To calculate the statistical significance of our results, we account for background events and errant dichroic mirror reflections as well as differences in the measured total and noise rates for the red or blue dichroic ports on each side, which yield unequal (biased) frequencies for various combinations of detector settings $a_{i} b_{j}$. Moreover, whereas we assume fair sampling (for both entangled and stellar photon detections) and fair coincidences for entangled photons [65], we adopt the conservative assumption that the local hidden-variable model could retain memory of settings and outcomes of previous trials [69-71]. As detailed in the Supplemental Material [57], we find that the measured fractions of corrupt coincidences are sufficiently low that the probability that a local hidden-variable model could explain the observed violations of Bell's inequality is $p \leq 1.78 \times 10^{-13}$ for experimental run 1 , and $p \leq 3.96 \times 10^{-33}$ for run 2 . These correspond to experimental violations of the $\mathrm{CHSH}$ bound by at least 7.31 and 11.93 standard deviations, respectively.

Conclusions.-For both runs, we assume fair sampling, close the locality loophole and, for the first time, explicitly constrain freedom of choice with astronomically chosen settings, relegating any local-realist models to have acted no more recently than $604 \pm 35$ and $577 \pm 40$ years ago, for runs 1 and 2, respectively. Therefore, any hiddenvariable mechanism exploiting the freedom-of-choice loophole would need to have been enacted prior to Gutenberg's invention of the printing press, which itself predates the publication of Newton's Principia by two and a half centuries. While a Bell test like ours only constrains a local-realist mechanism to have acted no later than the most recent of the astronomical emission events, we note that any process that requires both emission events to have been influenced by the same common cause would be relegated to even earlier times, to when the past light cones from each emission event intersected $2409 \pm 598$ and $4040 \pm$ 1363 years ago, for runs 1 and 2, respectively [72].

This work thus represents the first experiment to dramatically limit the space-time region in which hidden variables could be relevant, paving the way for future ground- and space-based tests with distant galaxies, quasars, patches of the cosmic microwave background (CMB), or other more exotic sources such as neutrinos and gravitational waves. Such tests could progressively push any viable hidden-variable models further back into deep cosmic history [1], billions of years in the case of quasars, back to the early universe in the case of the CMB, or even, in the case of primordial gravitational waves, further back to any period of inflation preceding the conventional big bang model [73-77].

The authors would like to thank Brian Keating, Michael J. W. Hall, Jan-Åke Larsson, Marissa Giustina, Ned Hall, Craig Callender, Jacob Barandes, David Kagan, and Larry Guth for useful discussions. Thanks to the Austrian National Bank (OENB) and the Bundesimmobiliengesellschaft (BIG) for providing the rooms for our receiving stations. This work was supported by the Austrian Academy of Sciences (OEAW), by the Austrian Science Fund (FWF) with SFB F40 (FOQUS) and FWF project CoQuS No. W1210-N16 and the Austrian Federal Ministry of Science, Research and Economy (BMWFW). A. S. F., D. I. K., A. H. G., and J. G. acknowledge support for this project from NSF INSPIRE Grant no. PHY-1541160. A. S. F. acknowledges support from NSF Grant No. SES-1056580. A. M. and I. S. acknowledge support from MIT's Undergraduate Research Opportunities Program (UROP). Portions of this work were conducted in MIT's Center for Theoretical Physics and supported in part by the U.S. Department of Energy under Contract No. DE-SC0012567. J. G. acknowledges support from NSF Grant No. PLR-1248097 and Harvey Mudd College.

*johannes.handsteiner@univie.ac.at
†asf@mit.edu
†anton.zeilinger@univie.ac.at

[1] J. Gallicchio, A. S. Friedman, and D. I. Kaiser, Testing Bell's Inequality with Cosmic Photons: Closing the SettingIndependence Loophole, Phys. Rev. Lett. 112, 110405 (2014).

[2] A. Einstein, B. Podolsky, and N. Rosen, Can quantummechanical description of physical reality be considered complete?, Phys. Rev. 47, 777 (1935).

[3] J. S. Bell, On the Einstein Podolsky Rosen Paradox, Physics 1, 195 (1964).

[4] S. J. Freedman and J. F. Clauser, Experimental Test of Local Hidden-Variable Theories, Phys. Rev. Lett. 28, 938 (1972).

[5] N. Brunner, D. Cavalcanti, S. Pironio, V. Scarani, and S. Wehner, Bell nonlocality, Rev. Mod. Phys. 86, 419 (2014).

[6] J.-Å. Larsson, Loopholes in Bell inequality tests of local realism, J. Phys. A 47, 424003 (2014).

[7] J. Kofler, M. Giustina, J.-A. Larsson, and M. W. Mitchell, Requirements for a loophole-free photonic Bell test using imperfect setting generators, Phys. Rev. A 93, 032115 (2016).

[8] A. Aspect, J. Dalibard, and G. Roger, Experimental Test of Bell's Inequalities Using Time-Varying Analyzers, Phys. Rev. Lett. 49, 1804 (1982).

[9] G. Weihs, T. Jennewein, C. Simon, H. Weinfurter, and A. Zeilinger, Violation of Bell's Inequality under Strict Einstein Locality Conditions, Phys. Rev. Lett. 81, 5039 (1998).

[10] P. M. Pearle, Hidden-variable example based upon data rejection, Phys. Rev. D 2, 1418 (1970).

[11] M. A. Rowe, D. Kielpinski, V. Meyer, C. A. Sackett, W. M. Itano, C. Monroe, and D. J. Wineland, Experimental 
violation of a Bell's inequality with efficient detection, Nature (London) 409, 791 (2001).

[12] D. N. Matsukevich, P. Maunz, D. L. Moehring, S. Olmschenk, and C. Monroe, Bell Inequality Violation with Two Remote Atomic Qubits, Phys. Rev. Lett. 100, 150404 (2008).

[13] M. Ansmann, H. Wang, R. C. Bialczak, M. Hofheinz, E. Lucero, M. Neeley, A. D. O'Connell, D. Sank, M. Weides, J. Wenner, A. N. Cleland, and J.M. Martinis, Violation of Bell's inequality in Josephson phase qubits, Nature (London) 461, 504 (2009).

[14] J. Hofmann, M. Krug, N. Ortegel, L. Gérard, M. Weber, W. Rosenfeld, and $\mathrm{H}$. Weinfurter, Heralded entanglement between widely separated atoms, Science 337, 72 (2012).

[15] M. Giustina, A. Mech, S. Ramelow, B. Wittmann, J. Kofler, J. Beyer, A. Lita, B. Calkins, T. Gerrits, S. W. Nam, R. Ursin, and A. Zeilinger, Bell violation using entangled photons without the fair-sampling assumption, Nature (London) 497, 227 (2013).

[16] B. G. Christensen, K. T. McCusker, J. Altepeter, B. Calkins, T. Gerrits, A. Lita, A. Miller, L. K. Shalm, Y. Zhang, S. W. Nam, N. Brunner, C. C. W. Lim, N. Gisin, and P. G. Kwiat, Detection-Loophole-Free Test of Quantum Nonlocality, and Applications, Phys. Rev. Lett. 111, 130406 (2013).

[17] B. Hensen, H. Bernien, A. E. Dréau, A. Reiserer, N. Kalb, M. S. Blok, J. Ruitenberg, R.F. L. Vermeulen, R. N. Schouten, C. Abellán, W. Amaya, V. Pruneri, M. W. Mitchell, M. Markham, D. J. Twitchen, D. Elkouss, S. Wehner, T. H. Taminiau, and R. Hanson, Loophole-free Bell inequality violation using electron spins separated by 1.3 kilometres, Nature (London) 526, 682 (2015).

[18] M. Giustina et al., Significant-Loophole-Free Test of Bell's Theorem with Entangled Photons, Phys. Rev. Lett. 115, 250401 (2015).

[19] L. K. Shalm et al., Strong Loophole-Free Test of Local Realism, Phys. Rev. Lett. 115, 250402 (2015).

[20] B. Hensen, N. Kalb, M. S. Blok, A. E. Dréau, A. Reiserer, R. F. L. Vermeulen, R. N. Schouten, M. Markham, D. J. Twitchen, K. Goodenough, D. Elkouss, S. Wehner, T. H. Taminiau, and R. Hanson, Loophole-free Bell test using electron spins in diamond: second experiment and additional analysis, Sci. Rep. 6, 30289 (2016).

[21] W. Rosenfeld, D. Burchardt, R. Garthoff, K. Redeker, N. Ortegel, M. Rau, and H. Weinfurter, Event-ready Bell-test using entangled atoms simultaneously closing detection and locality loopholes, arXiv:1611.04604.

[22] J. S. Bell, The Theory of Local Beables, Epistemological Lett. 9, 86 (1976).

[23] A. Shimony, M. A. Horne, and J. F. Clauser, Comment on 'The theory of local beables', Epistemological Lett. 13, 97 (1976).

[24] J. S. Bell, Free variables and local causality, Epistemological Lett. 15, 79 (1977).

[25] C. H. Brans, Bell's theorem does not eliminate fully causal hidden variables, Int. J. Theor. Phys. 27, 219 (1988).

[26] J. Kofler, T. Paterek, and Č. Brukner, Experimenter's freedom in Bell's theorem and quantum cryptography, Phys. Rev. A 73, 022104 (2006).
[27] M. J. W. Hall, Local Deterministic Model of Singlet State Correlations Based on Relaxing Measurement Independence, Phys. Rev. Lett. 105, 250404 (2010).

[28] M. J. W. Hall, Relaxed Bell inequalities and KochenSpecker theorems, Phys. Rev. A 84, 022102 (2011).

[29] J. Barrett and N. Gisin, How Much Measurement Independence Is Needed to Demonstrate Nonlocality?, Phys. Rev. Lett. 106, 100406 (2011).

[30] D. E. Koh, M. J. W. Hall, Setiawan, J. E. Pope, C. Marletto, A. Kay, V. Scarani, and A. Ekert, Effects of Reduced Measurement Independence on Bell-Based Randomness Expansion, Phys. Rev. Lett. 109, 160404 (2012).

[31] M. Banik, M. Rajjak Gazi, S. Das, A. Rai, and S. Kunkri, Optimal free will on one side in reproducing the singlet correlation, J. Phys. A 45, 205301 (2012).

[32] R. Colbeck and R. Renner, Free randomness can be amplified, Nat. Phys. 8, 450 (2012).

[33] L. P. Thinh, L. Sheridan, and V. Scarani, Bell tests with minentropy sources, Phys. Rev. A 87, 062121 (2013).

[34] G. Pütz, D. Rosset, T. J. Barnea, Y.-C. Liang, and N. Gisin, Arbitrarily Small Amount of Measurement Independence Is Sufficient to Manifest Quantum Nonlocality, Phys. Rev. Lett. 113, 190402 (2014).

[35] G. Pütz and N. Gisin, Measurement dependent locality, New J. Phys. 18, 055006 (2016).

[36] M. J. W. Hall, The significance of measurement independence for Bell inequalities and locality, in At the Frontier of Spacetime-Scalar-Tensor Theory, Bell's Inequality, Mach's Principle, Exotic Smoothness, edited by T. Asselmeyer-Maluga (Springer, Switzerland, 2016), Chap. 11, p. 189.

[37] S. Pironio, Random 'choices' and the locality loophole, arXiv: 1510.00248.

[38] T. Scheidl, R. Ursin, J. Kofler, S. Ramelow, X. S. Ma, Th. Herbst, L. Ratschbacher, A. Fedrizzi, N. K. Langford, T. Jennewein, and A. Zeilinger, Violation of local realism with freedom of choice, Proc. Natl. Acad. Sci. U.S.A. 107, 19708 (2010).

[39] D. Aktas, S. Tanzilli, A. Martin, G. Pütz, R. Thew, and N. Gisin, Demonstration of Quantum Nonlocality in the Presence of Measurement Dependence, Phys. Rev. Lett. 114, 220404 (2015).

[40] J. Barrett, L. Hardy, and A. Kent, No Signaling and Quantum Key Distribution, Phys. Rev. Lett. 95, 010503 (2005).

[41] S. Pironio, A. Acín, N. Brunner, N. Gisin, S. Massar, and V. Scarani, Device-independent quantum key distribution secure against collective attacks, New J. Phys. 11, 045021 (2009).

[42] U. Vazirani and T. Vidick, Fully Device-Independent Quantum Key Distribution, Phys. Rev. Lett. 113, 140501 (2014).

[43] S. Pironio, A. Acín, S. Massar, A. B. de La Giroday, D. N. Matsukevich, P. Maunz, S. Olmschenk, D. Hayes, L. Luo, T. A. Manning, and C. Monroe, Random numbers certified by Bell's theorem, Nature (London) 464, 1021 (2010).

[44] R. Gallego, L. Masanes, G. de la Torre, C. Dhara, L. Aolita, and A. Acín, Full randomness from arbitrarily deterministic events, Nat. Commun. 4, 2654 (2013). 
[45] One may even consider retrocausal models in which the relevant hidden variable affects the measurement settings from the future $[46,47]$. The point is that the relevant hidden variable may be associated with spacetime regions far removed from the source of entangled particles.

[46] O. Costa de Beauregard, S-matrix, Feynman zigzag, and Einstein correlation, Phys. Lett. A 67, 171 (1978).

[47] H. Price and K. Wharton, Disentangling the Quantum World, Entropy 17, 7752 (2015).

[48] We note that it is only possible to fully close the locality and freedom-of-choice loopholes by making certain assumptions. If settings were fully determined at the beginning of the universe, they could always be known at the distant measurement locations or be correlated with the hidden variables.

[49] J.S. Bell and B. d'Espagnat, Thinkshop in Physics: Experimental Quantum Mechanics, Erice, 1976, Prog. Sci. Culture 1/4, 439 (1976).

[50] T. Maudlin, Quantum Non-Locality and Relativity: Metaphysical Intimations of Modern Physics (Wiley, New York, 1994).

[51] L. Vaidman, Tests of Bell inequalities, Phys. Lett. A 286, 241 (2001).

[52] T. Kim, M. Fiorentino, and F. N. C. Wong, Phase-stable source of polarization-entangled photons using a polarization Sagnac interferometer, Phys. Rev. A 73, 012316 (2006).

[53] A. Fedrizzi, T. Herbst, A. Poppe, T. Jennewein, and A. Zeilinger, A wavelength-tunable fiber-coupled source of narrowband entangled photons, Opt. Express 15, 15377 (2007).

[54] J. A. Cardelli, G. C. Clayton, and J. S. Mathis, The relationship between infrared, optical, and ultraviolet extinction, Astrophys. J. 345, 245 (1989).

[55] E. L. Fitzpatrick, Correcting for the effects of interstellar extinction, Publ. Astron. Soc. Pac. 111, 63 (1999).

[56] E. F. Schlafly and D. P. Finkbeiner, Measuring reddening with Sloan Digital Sky Survey stellar spectra and recalibrating SFD, Astrophys. J. 737, 103 (2011).

[57] See Supplemental Material at http://link.aps.org/ supplemental/10.1103/PhysRevLett.118.060401 for more information about the experimental setup and data analysis, which includes Refs. [58-60].

[58] C. Fröhlich and G. E. Shaw, New determination of Rayleigh scattering in the terrestrial atmosphere, Appl. Opt. 19, 1773 (1980).

[59] D. Elkouss and S. Wehner, (Nearly) optimal P values for all Bell inequalities, NPJ Quantum Information 2, 16026 (2016).

[60] W. Hoeffding, Probability inequalities for sums of bounded random variables, J. Am. Stat. Assoc. 58, 13 (1963).

[61] A. Berk, L. S. Bernstein, and D. C. Robertson, Space Science Instrumentation, Technical Report (1987).

[62] J. A. Stone and J. H. Zimmerman, Index of refraction of air, Engineering Metrology Toolbox, National Institute of
Science and Technology, (Gaithersburg, Maryland 2011), http://emtoolbox.nist.gov/Wavelength/Documentation.asp.

[63] M. A. C. Perryman, L. Lindegren, J. Kovalevsky, E. Hoeg, U. Bastian, P. L. Bernacca, M. Crézé, F. Donati, M. Grenon, M. Grewing, F. van Leeuwen, H. van der Marel, F. Mignard, C. A. Murray, R. S. Le Poole, H. Schrijver, C. Turon, F. Arenou, M. Froeschlé, and C.S. Petersen, The HIPPARCOS catalogue, Astron. Astrophys. 323, L49 (1997).

[64] F. van Leeuwen, Validation of the new Hipparcos reduction, Astron. Astrophys. 474, 653 (2007).

[65] J.-Å. Larsson, M. Giustina, J. Kofler, B. Wittmann, R. Ursin, and S. Ramelow, Bell-inequality violation with entangled photons, free of the coincidence-time loophole, Phys. Rev. A 90, 032107 (2014).

[66] T. Scheidl, R. Ursin, A. Fedrizzi, S. Ramelow, X.-S. Ma, T. Herbst, R. Prevedel, L. Ratschbacher, J. Kofler, T. Jennewein, and A. Zeilinger, Feasibility of $300 \mathrm{~km}$ quantum key distribution with entangled states, New J. Phys. 11, 085002 (2009).

[67] J. F. Clauser, M. A. Horne, A. Shimony, and R. A. Holt, Proposed Experiment to Test Local Hidden-Variable Theories, Phys. Rev. Lett. 23, 880 (1969).

[68] S. Popescu and D. Rohrlich, Quantum nonlocality as an axiom, Found. Phys. 24, 379 (1994).

[69] R. D. Gill, Time, Finite Statistics, and Bell's Fifth Position, in Proceedings of Foundations of Probability and Physics2, Math. Modelling in Phys., Engin., and Cogn. Sc., Vol. 5 (Växjö University Press, 2003), p. 179.

[70] R. D. Gill, Statistics, causality, and Bell's theorem, Stat. Sci. 29, 512 (2014).

[71] P. Bierhorst, A robust mathematical model for a loopholefree Clauser-Horne experiment, J. Phys. A 48, 195302 (2015).

[72] A. S. Friedman, D. I. Kaiser, and J. Gallicchio, The shared causal pasts and futures of cosmological events, Phys. Rev. D 88, 044038 (2013).

[73] A. H. Guth, Inflationary universe: A possible solution to the horizon and flatness problems, Phys. Rev. D 23, 347 (1981).

[74] A. D. Linde, A new inflationary universe scenario: A possible solution of the horizon, flatness, homogeneity, isotropy and primordial monopole problems, Phys. Lett. B 108, 389 (1982).

[75] A. Albrecht and P. J. Steinhardt, Cosmology for grand unified theories with radiatively induced symmetry breaking, Phys. Rev. Lett. 48, 1220 (1982).

[76] A.H. Guth and D. I. Kaiser, Inflationary cosmology: Exploring the Universe from the smallest to the largest scales, Science 307, 884 (2005).

[77] A. H. Guth, D. I. Kaiser, and Y. Nomura, Inflationary paradigm after Planck 2013, Phys. Lett. B 733, 112 (2014). 